IDEAs IN ECOLGGY AND EVRLUTION 7: 1-2, 2014

doi:10.4033/iee.2014.7.1.e

(C) 2014 The Author. (C) Ideas in Ecology and Evolution 2014

Received 12 January 2014; Accepted 16 January 2014

\title{
Editorial
}

\section{Self-citation by researchers: narcissism or an inevitable outcome of a cohesive and sustained research program?}

\author{
Steven J. Cooke and Michael R. Donaldson \\ Steven J. Cooke (steven_cooke@carleton.ca), Fish Ecology and Conservation Physiology Laboratory, Department \\ of Biology and Institute of Environmental Science, Carleton University, Ottawa, Ontario, Canada
}

Michael R. Donaldson (michael.r.donaldson@gmail.com), Department of Natural Resources and Environmental Sciences, University of Illinois, Urbana, Illinois, USA

The research community remains focused with enumerating, evaluating, and ranking the research productivity of individual authors despite the apparent shortcomings of doing so. Basic yet widely used citation metrics such as "total cites" (Adam 2002) or "Hirsch (h) index" (Hirsch 2005) require a count of the number of times that a given author's works are cited. Fortunately there are a variety of electronic bibliometric tools (e.g., Web of Science, Google Scholar, Scopus) that do that work for us. Interestingly, those tools tend to generate default counts that can include self-citations. Selfcitations can be defined as occurrences in which the citing and cited papers share at least one author in common (Asknes 2003), although various definitions have been proposed (Fowler and Aksnes 2007, Costas et al. 2010). Self-citations can be easily filtered out with a few clicks to generate "corrected" indices (e.g., Schreiber 2007, Brown 2009) or those that discount self-cites (e.g., Ferrara and Romero 2013), but is it necessary to do so? Here, we argue that self-citations need not necessarily be considered a form of narcissistic behavior, and instead could be indicative of a cohesive research program, in which authors refer to their previous relevant works in order to enhance their subsequent contributions to knowledge.

When applying for scientific positions, promotions, tenure, or awards, one must decide whether they will report their "productivity" with or without self-citations, or include both. And, those assessing such researchers must decide which they wish to consider and whether they will "penalize" someone that fails to exclude selfcitations. Some individuals may feel that it is abhorrent to include self-citations while others may be indifferent.
On the surface, "self-citation" may appear to border on narcissism. However, the argument could also be made that self-citation is in fact an indicator of one's prominence and productivity in their field. Consider a researcher with a focused research program publishing year after year on related topics, with papers building upon ideas and discoveries codified in previous work. One would expect significant reliance on research papers from the same research lab. Indeed, is that not what an "ideal" research program should look like? Similarly, if one is working in a highly specialized field where there is simply little other research effort, self-citation would be essential. The more productive one is in terms of output in quantity of papers would also inherently lead to greater potential for self-citations. In this sense, it is reasonable to think that self-citation itself could be used as an indicator of the extent to which one has a cohesive and coordinated research program, with the extent of self-citation scaling with extent of output (in number of papers) from a research program.

When building a research program, self-citations can be an important aspect of developing a cohesive knowledge base and moving science forward. For example, if a research program has already been established, either by the author themselves or their collaborators and co-authors, it follows that self-citations would be necessary to develop the rationale that the current work is building on previously accumulated knowledge. Likewise, when interpreting findings by drawing on existing literature, self-citations are often necessary. For example, depending on the field of study and research questions being asked, the existing literature may be predominated by the author's own 
research team, requiring self-citations (e.g., emerging fields of study like molecular ecology). Thus, selfcitations can serve the dual purpose of providing rationale for the present work and also incrementing the knowledge provided by existing work. In this sense, self-citation could indicate a cohesive research program. A unified body of knowledge could in turn influence others (Donaldson and Cooke 2014). Indeed, Fowler and Aksnes (2007) show that the more one cites oneself, the more one is cited by others.

There are certainly instances where self-citation would be considered problematic. The potential for egregious self-citations exists (i.e., authors that go out of their way to cite their own work, even if only tangentially related), the onus for avoiding the manipulation of self-citations falls on the authors, or at the very least is something that should be corrected at the peer-review stage, prior to publication. There are also a number of strategies for identifying egregious attempts to manipulate bibliometrics through self-citation analysis (Bartneck and Kokkelmans 2011). Likewise, a researcher could intentionally cite their own work while failing to consider the broader literature. Such behaviour would be especially egregious if the research area is well developed. In the most insidious cases this could be done by researchers to directly manipulate their citation metrics (Testa 2008). However, it could also be done by a naïve researcher and simply reflect a lack of familiarity with relevant literature. Yet, conversely, failing to cite one's own work if and when it is the most relevant reference is no different than not citing someone else's key paper. That is, intentionally avoiding self-citation should not be the norm.

Trying to discern whether individual self-citation patterns are legitimate is probably best achieved at the level of an individual paper as judged by knowledgeable referees and astute, experienced editors (Cooke and Lapointe 2012). Has the author used a diversity of references, paying homage to the classic foundational work while simultaneously including contemporary references? Extensive self-citation may not be inherently wrong if the referees and editor judge that the references used (both self-citations and others) are indeed the most relevant references. If the scientific community serves as a filter to unwarranted self-citations during the peer review process, then we argue that self-citations-no matter the extent - are relevant and should be included as overall measures of a researcher's productivity and influence in that it is an inevitable outcome of a coordinated, sustained and productive research program.

\section{References}

Adam, D. 2002. Citation analysis: The counting house. Nature 415: 726-729. CrossRef

Aksnes, D.W. 2003. A macro study of self-citation. Scientometrics 56: 235-246. CrossRef

Bartneck, C., and S. Kokkelmans. 2011. Detecting hindex manipulation through self-citation analysis. Scientometrics 87: 85-98. CrossRef

Brown, R.J.C. 2009. A simple method for excluding self-citation from the h-index: the b-index. Online Information Review 33: 1129-1136. CrossRef

Cooke, S.J., and N.W.R. Lapointe. 2012. Addressing editor(ial) malpractice in scientific journals. Ideas in Ecology and Evolution 5:84-92. CrossRef

Costas, R., van Leeuwen, T.N., and M. Bordons. 2010. Self-citations at the meso and individual levels: effects of different calculation methods Scientometrics 82: 517-537. CrossRef

Donaldson, M.R. and S.J. Cooke. 2014. Scientific publications: Moving beyond quality and quantity toward influence. BioScience 64: 12-13.

Ferrara, E., and A.E. Romero. 2013. Scientific impact evaluation and the effect of self-citations: Mitigating the bias by discounting the h-index. Journal of the American Society of Information Science 64: 23322339. CrossRef

Fowler, J.H., and D.W. Aksnes. 2007. Does self-citation pay? Scientometrics. 72: 427-437. CrossRef

Hirsch, J.E. 2005. An index to quantify an individual's scientific research output. Proceedings of the National Academy of Sciences of the United States of America 102: 16569-16572. CrossRef

Schreiber, M. 2007. Self-citation corrections for the Hirsch index. Europhysics Letters 78: 30002. CrossRef

Testa, J. 2008. Playing the system puts self-citation's impact under review. Nature 455: 729. $\underline{\text { CrossRef }}$ 